\title{
Language Strategies to Reduce Anxiety of At-risk Children Deprived of Parental Care
}

\author{
Katerina I. Zlatkova-Doncheva ${ }^{1, *}$ \\ ${ }^{1}$ Faculty of Education, St Cyril and Methodius University of Veliko Tarnovo, Bulgaria \\ "Correspondence: Faculty of Education, St Cyril and Methodius University of Veliko Tarnovo, Bulgaria. Tel: \\ 359-876-563-679. E-mail: kzlatkova@gmail.com
}

Received: September 3, 2019

Accepted: October 6, 2019 Online Published: October 25, 2019

doi:10.5430/wje.v9n5p100

URL: https://doi.org/10.5430/wje.v9n5p100

\begin{abstract}
The present study examines language impact on anxiety in at-risk children deprived of parental care. Bulgarian children without parents $(\mathrm{n}=40)$ divided into 3 age groups (aged 7-10; aged 11-14; and aged 15-17) embed intervention accomplished by four volunteers using four interaction strategies: normal voice and positive language; high tone and positive language; normal tone and negative language, and high tone and negative language. Surveillance has been conducted subjecting anxiety reactions of children measured in 10 indicators: diffidence, dependence, dissatisfaction, reliance, insecurity (for self-assessment anxiety); inadequacy, inactivity, non-communication, inability to seek help, and lack of empathy (for interpersonal anxiety). The present study results demonstrate that linguistic signs have higher influence than paralinguistic cues on children's behaviour and the use of negative language would increase anxiety in children to a greater extent when the tone is normal while raising the tone would enhance self-assessment and interpersonal anxiety of children with emotional disorders. Balanced use of negative and positive language combination with a different tone in different situations would increase social functioning of the child. The use of different strategies for interaction by specialists - with normal and high tone, encouragement or reprimand should be tailored to the specifics of the child as well as to the relevant skills to be developed.
\end{abstract}

Keywords: at-risk children, anxiety, children deprived of parental care, social skills, language, paralinguistic signs, lexical content

\section{Introduction}

The term "at-risk children" applies to children having limited reading proficiency, experienced abuse or trauma, having a disability or illness, or having shown behavior problems according to risk factors - family, community and child itself and refers to long-term deficits, such as school failure, behavior problems, or incarceration (Rak, 1996; Moore, 2006, Zlatkova-Doncheva, 2018). Children deprived of parental care are identified as a highly vulnerable group with the highest risk and often determined with emotional and behavioral problems. Their specifics descend of social background as well as mental, emotional and physical development insufficiency.

High levels of anxiety, emotional disorders, pro-social behavior shortages and low communication and social skills apply as basic features of at-risk children deprived of parental care steaming from the social situation they are in, lack of a role-based parenting model and emotional acceptance which negatively affects their overall mental and physical development and places them in less favorable conditions and inequality with their peers (Zlatkova-Doncheva, 2018). At-risk children deprived of parental care specifics imply a serious, purposeful and specific approach of work that creates opportunities for overcoming shortages which include reducing anxiety to help personal development.

\section{Material Studied}

\subsection{At-risk Children Deprived of Parental Care Definitions}

At-risk children definitions are summarized as children who experience intense risk factors that prevent them from 
reaching their full potential (Moore, 2006). Children deprived of parental care are one of the most vulnerable at-risk groups living in a specialized institution or residence service, due to a permanent lack of parental care, and have an unfavorable life perspective because of their family, the community, personality accents, and often have negative impact in regard to their personal development, health, mental and emotional status, socialization, functioning and adaptation in the society (Kuzmanova-Kartalova, 2013; Kuteva - Tsvetkova, 2000; Georgiev, 2019; Dimitrova, 2016, Zdravkova, 2015). Numerous studies define children at risk deprived of parental care as children raised without parents; children who live in institutional care; children left without the custody of their parents; with parents missing, deprived of parental rights or having limited rights; not permanently caring for their children or permanently being unable to raise them; having created danger to their physical, mental, moral, intellectual and social development, or having experienced forms of violence and abuse against their children (Zlatkova-Doncheva, 2018).

The attachment concept comes from the attachment theory, formulated by psychiatrist and psychoanalyst John Bowlby, as a result of his focused experimental and research work on the effect of separation between parent and child, which influences the individual's overall personal and behavioral development. Bowlby creates a psychological, evolutionary, and ethological theory that provides a descriptive and explanatory framework for understanding the interpersonal relationships of human beings. His followers, the attachment theorists, believe that children need a secure relationship with the adults taking care of them, without which a normal social and emotional development is disrupted. At-risk children deprived of parental care usually have a broken relationship with their parents and their personal features could be largely explained through the attachment theory (Zlatkova-Doncheva, 2017; Stamatov, 2000: 83-95). Ambivalent and unreliable attachment concepts arise from the attachment theory and explain the separation from the parent affecting the child emotionally. Children develop high levels of anxiety when mothers have not had physical contact and rejected them as a result of separation (unreliable attachment), as well as when mothers give controversial emotional feedback - rejection alternates with acceptance or the child has experienced periodic shorter or longer partitions with the mother (ambivalent attachment). In both cases, the separation experience identifies two major emotional states: anxiety and anger underlining personality such as aggression, adaptation problems, communication, self-assessment anxiety, and interpersonal anxiety (Zlatkova-Doncheva, 2017; Stamatov, 2000). Building a "basic trust" is an existential expression of attachment that lies in the child's inner conviction of security and trust, stemming from the quality of the mother's presence, emotional attachment, and responsiveness to the child's needs and care. The concept was formulated by Eric Erickson and has been complemented by the theoretical propositions of Erich From related to the identification of the individual's existential needs - the need for connection with others, care and responsibility for others, inner significance, safety, identity and values (Zlatkova-Doncheva, 2018). This term is used in the context of at-risk children deprived of parental care specifics defining them with compromised basic trust, unable to connect emotionally, to empathize with others and to communicate adequately.

\subsection{Anxiety Definitions}

Different theories about anxiety have been developed by various studies. Anxiety has also been recognized as an important factor for understanding as a foundation of interpersonal behavior and psychological functioning (Greca \& Lopez, 1998; Leary, 1983). Other theories identify anxiety with fear and distinction between both terms is based on presence or absence of cues. Fear is defined as a reaction to an obvious danger while anxiety is determined as a subjective apprehension. Therefore, anxiety is observed as fear with "hidden cues" in the personality of an individual (Barlow, 2002). A. M. Prihozhan determines anxiety as an obvious or hidden dependence of the individual on external stimuli, insecurity, and affirmation of personality through appreciation of others due to traumatic or negative emotional experiences. Prihojan points out that anxiety can be categorized as self-assessment anxiety and interpersonal anxiety that is directly dependent on aggressive behavior in different situations, which in itself is brought out by suppressed anxiety. Anxiety as an expression also determines the way in which an individual interacts with others and adapts to the outside world, which has a direct connection with the development of certain social skills - communication, independence, decision-making skills, adequate self-esteem, emotional resilience and confidence (Zlatkova-Doncheva, 2018, Prihojan, 2000). The connection with the attachment theory appears in order to determine the anxiety factors as inability of the child without parental care to find an internal incentive to affirm self in front of others and obstacles to connect emotionally which is assigned by weak trusting skills of the child due to unreliable or ambivalent attachment and lack of basic confidence.

\subsection{Linguistic and Paralinguistic Signs}

Various studies emphasize the role of language to children's behavior; children are sensitive to linguistic and paralinguistic elements of the language from early childhood (Clarkson \& Clifton, 1985). However, various authors 
digest the use of different paralinguistic styles with higher impact on reactions of the child (Kitamura and Burnham, 2003; Kitamura and Lam, 2009). Other concepts give greater significance on influencing child's behavior towards lexical content than paralinguistic cues and outline greater importance to what is said to them than how it is said (Friend, 2003). M. Friend develops the assumption that the meaning of words plays a more important regulatory role in order to influence a child's behavior. Her study determines the lexical content advantage, and is relevant to targeting in behavioral aspect (Friend, 2000; Friend \& Bryant, 2000; Friend, 2003; Morton \& Trehub 2001). Other research studies have also investigated children's behavior being influenced by linguistic rather than paralinguistic signs (Doncheva, 2019; Friend \& Bryant, 2000; Hancock et al., 2000; Moore et al., 1993; Solomon \& Ali, 1972).

The influence of paralinguistic cues and linguistic signs should also be assumed according to some additional issues as the emotional, social, behavioral and personal situation of the child. It is not apparent to display the same role of language used to children with different situations including disorders, accentuation, personal traits as well as social background. Children's interpretation of messages is different for children with emotional disorders and they often express inadequacy in the perception of different communication channels. Lexical content impact is likely to be limited to a certain combination of content response and children with emotional-behavioral disorders identify messages in more positive emotional meaning than normal children (Friend, Becker, 1987). Providing more acceptable assumptions about how language could influence anxiety in children at risk is recommended to be developed in further research and is one of the tasks of this study.

\section{Problem Statement and Area Description}

The present study is aimed at investigating possible strategies influencing self-assessment and interpersonal anxiety (Prihojan, 2000) of at-risk children deprived of parental care. Due to their specifics, personal features and behavioral characteristics they are placed in more vulnerable position than their peers and justify the need of different approach to compensate the negative impact on their overall development. The hereby proposed scope is an instance from Bulgaria $(N=40)$ and it includes at-risk children grown outside of their families living in residential care centers. The general hypothesis of the study underlines that the use of language and specific language strategies for interaction would affect anxiety of children at-risk deprived of parental care.

The question of the present research is: What is the impact of language to self- assessment anxiety and interpersonal anxiety of at-risk children deprived of parental care and would negative language with high tone increase anxiety among children?

\section{Methodology}

The present study is aimed to research language impact on anxiety in at-risk children raised outside of their families. The use of lexical signs (positive and negative words) and a paralinguistic cue (tone as a power of the voice) has been experienced as intervention. General hypothesis underlines that negative language with high tone would have higher impact on anxiety in children in order to increase self-assessment anxiety as well as interpersonal anxiety.

An intervention group consisting of children at risk without parents living across Bulgarian residential centers $(N=40)$ experienced four different interaction strategies applied by four volunteers: normal voice and positive language; high tone and positive language; normal tone and negative language, and high tone and negative language. Surveillance has been conducted for data collection accomplished by independent observer subjecting reactions of children based on A. M. Prihojan's anxiety concepts of defining anxiety in the context of self-assessment and interpersonal anxiety (Prihojan, 2000, Zlatkova-Doncheva, 2018). The criteria are measured in the following 10 indicators: diffidence, dependence, dissatisfaction, reliance, insecurity (for self-assessment anxiety); inadequacy, inactivity, non-communication, inability to seek help, and lack of empathy (for interpersonal anxiety). Dependent variables were defined according to possibility to measure certain behavior reactions indicating anxiety. The measurement item analysis registers high internal consistency in general, positive correlation and high reliability $\left(r_{s h}=0.685\right.$, alpha of Crohn $=\alpha=\alpha=$.734). The items' difficulties are within acceptable limits and the scale has a high reliability value with an average inter-correlation of 0.368 .

Participants from 4 residential care homes from the town of Veliko Tarnovo were divided into three age groups: aged 7-10 (primary school, $N=11$ ), aged 11-13 (lower secondary school age group, $N=12$ ) and aged 14-17 (secondary school age group $(N=17) .70 \%$ were female and $30 \%$ were male. 


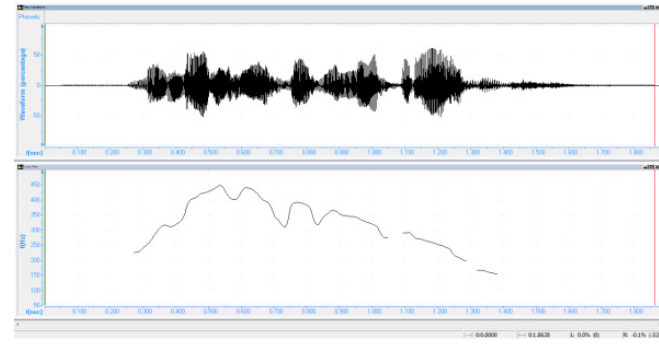

Figure 1. High Tone

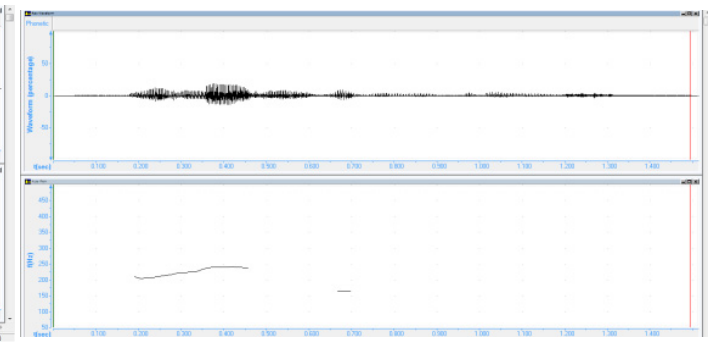

Figure 2. Normal Tone

Use of the tone (power of the voice) is preliminary measured and all linguistic signs (positive and negative words) are used with a normal tone (sound of $200 \mathrm{~Hz}$ ) and with a high tone $-450 \mathrm{~Hz}$. (Figure 1 and Figure 2).

Conducted surveillance of the anxiety indicators used the following intervention strategies:

Table 1. Measurements

\begin{tabular}{|c|c|c|c|c|c|}
\hline Behavior reactions & Indicator & $\begin{array}{l}\text { Positive } \\
\text { language } \\
\text { Normal tone }\end{array}$ & $\begin{array}{l}\text { Positive } \\
\text { language High } \\
\text { tone }\end{array}$ & $\begin{array}{l}\text { Negative } \\
\text { language } \\
\text { Normal tone }\end{array}$ & $\begin{array}{l}\text { Negative } \\
\text { language } \\
\text { High tone }\end{array}$ \\
\hline & & Yes1/No 0 & Yes1/No 0 & Yes1/No 0 & Yes1/No 0 \\
\hline Gives up when difficult & Diffidence & $1 / 0$ & $1 / 0$ & $1 / 0$ & $1 / 0$ \\
\hline Seeks continuous approval & Dependence & $1 / 0$ & $1 / 0$ & $1 / 0$ & $1 / 0$ \\
\hline $\begin{array}{l}\text { Is frustrated when does not receive positive } \\
\text { evaluation }\end{array}$ & Dissatisfaction & $1 / 0$ & $1 / 0$ & $1 / 0$ & $1 / 0$ \\
\hline Cannot work independently & Reliance & $1 / 0$ & $1 / 0$ & $1 / 0$ & $1 / 0$ \\
\hline Cannot make decisions in small tasks & Insecurity & $1 / 0$ & $1 / 0$ & $1 / 0$ & $1 / 0$ \\
\hline $\begin{array}{l}\text { Wants to attract attention in an inapropriate } \\
\text { way }\end{array}$ & Inadequacy & $1 / 0$ & $1 / 0$ & $1 / 0$ & $1 / 0$ \\
\hline Does not take initiative to do things & Inactivity & $1 / 0$ & $1 / 0$ & $1 / 0$ & $1 / 0$ \\
\hline $\begin{array}{l}\text { Takes other people's stuff without asking } \\
\text { for permission }\end{array}$ & Non-communication & $1 / 0$ & $1 / 0$ & $1 / 0$ & $1 / 0$ \\
\hline $\begin{array}{l}\text { Does not know how to seek help when } \\
\text { difficult }\end{array}$ & Inability to seek help & $1 / 0$ & $1 / 0$ & $1 / 0$ & $1 / 0$ \\
\hline Does not want to help mates & Lack of empathy & $1 / 0$ & $1 / 0$ & $1 / 0$ & $1 / 0$ \\
\hline
\end{tabular}

Result proceedings from the experienced four strategies compare means between all the participants of the residential centers and a second intervention group $(N=11)$ has been defined randomly. Repetitive measures in a normal environment (their daily life) have been accomplished for the new intervention group to check registered significance of self-assessment anxiety and interpersonal anxiety and whether the results are affected by the experimental impact strategies rather than caused by personality traits or accents in the child's character. The same 10 anxiety indicators are verified in an assessment scale replenished by specialists and care givers who normally look after children: diffidence, dependence, dissatisfaction, reliance, insecurity (for self-assessment anxiety); inadequacy, inactivity, non-communication, inability to seek help, and lack of empathy (for interpersonal anxiety).

All indicators were measured by the same observed behavior reactions showing expression of self-assessment anxiety and interpersonal anxiety variables that have been experienced within the surveillance (see table 1). The assessment rating scale contains the same five behavioral expressions of anxiety from 1 to 4, where 1 is "no, never", 2 - "seldom", 3 - "yes, sometimes", 4 - "yes, often".

Reduction of the limitations has been completed throughout detailed evaluation by the specialists and care givers who work and have daily contact with the child. For this purpose every child of the second intervention group has been assessed by six specialists and means of all grades have been formed to determine objectivity of results. 


\section{Results}

Mean values register increase of anxiety in children within the use of negative language in both the normal and high tone of the voice leading to a presumption of the strongest impact of linguistic signs (words) to paralinguistic (tone) cues (table 2). The results confirm the thesis of M. Friend about the leading role of lexical signs to paralinguistic cues due to several research studies (Friend, 2000).

Table 2. Mean Values of 4 Strategies Used

\begin{tabular}{lcccccccc}
\hline & $\begin{array}{l}\text { Positive } \\
\text { Normal tone }\end{array}$ & language & $\begin{array}{l}\text { Positive } \\
\text { High tone }\end{array}$ & $\begin{array}{l}\text { language } \\
\text { language } \\
\text { Normal tone }\end{array}$ & $\begin{array}{l}\text { Negative } \\
\text { High tone }\end{array}$ \\
\hline Diffidence & Mean & SD & Mean & SD & Mean & SD & Mean & SD \\
Dependence & 0.17 & 0.38 & 0.10 & 0.30 & 0.60 & 0.50 & 0.63 & 0.49 \\
Dissatisfaction & 0.20 & 0.40 & 0.15 & 0.36 & 0.67 & 0.47 & 0.60 & 0.50 \\
Reliance & 0.00 & 0.00 & 0.20 & 0.40 & 0.85 & 0.36 & 0.85 & 0.36 \\
Insecurity & 0.07 & 0.27 & 0.12 & 0.33 & 0.55 & 0.50 & 0.48 & 0.51 \\
Inadequacy & 0.07 & 0.27 & 0.07 & 0.27 & 0.70 & 0.46 & 0.62 & 0.49 \\
Inactivity & 0.17 & 0.38 & 0.15 & 0.36 & 0.45 & 0.50 & 0.65 & 0.48 \\
Non-communication & 0.22 & 0.42 & 0.12 & 0.33 & 0.77 & 0.42 & 0.60 & 0.50 \\
Inability to seek help & 0.10 & 0.30 & 0.05 & 0.22 & 0.32 & 0.47 & 0.30 & 0.46 \\
Lack of empathy & 0.07 & 0.26 & 0.07 & 0.27 & 0.82 & 0.38 & 0.65 & 0.48 \\
\hline
\end{tabular}

As shown in Table 2, negative language with normal tone of voice registers the highest results of all self-assessment anxiety variables within over half shares of the intervention group. More than half of the children register interpersonal anxiety increasing within 4 out of 5 indicators (inadequacy, inactivity, non-communication, inability to seek help, and lack of empathy).

Most children register interpersonal anxiety in negative language with normal tone of voice within the lack of empathy variable. A large scope of children registers increase in the same variable within high tone of voice. Dissatisfaction variable increases its level with the use of negative language with both high and normal tone of voice. In addition, dependence of external stimulus as well as inactivity variables reported relatively high values in all four strategies: positive and negative language with high and normal tone, which could lead to the assumption that anxiety of the intervention group, could be a personal trait quality.

Four strategies influencing anxiety variables are investigated within a correlation analysis demonstrating the best results between dependence and dissatisfaction when positive language with high tone is used $(r=.84 ; p<.001)$, and reliance and insecurity variables are also well-matched with the same strategy $(r=0.75 ; p<.001)$. Present data confirm the assurance that anxiety should be considered as a personal quality of the intervention group rather than an effect of the four strategies impact. Similar trend has been registered within interpersonal anxiety indicators where diffidence and dependence variables reported best correlation in the use of positive language with normal tone $(r$ $=.70 ; p<.001)$. Inability to seek help with a positive language and high tone demonstrates good correlation between the lack of empathy and positive language with normal tone $(r=.64 ; p<.001)$, and inability to seek help in the use of positive language with normal tone has a good match with reliance in the use of negative language with normal tone $(r=.64 ; p<.001)$. Negative language with high tone has a high impact on inactivity reporting good correlation with reliance $(r=.57 ; p<.001)$ and insecurity $(r=.53 ; p<.001)$. Positive language with normal tone also influences inactivity interacting very good with diffidence $(\mathrm{r}=.54 ; p<.001)$ and dependence $(\mathrm{r}=.45 ; p<.001)$.

Data analysis suggests that the activity inclusion of the child as well as the independence from external stimulus, confidence and security in oneself is more affiliated with their personality rather than affected by the four intervention strategies. In this sense, it could be assumed that the extent of child's activity involvement rather depends on anxiety as a personal trait, and is not that influenced by the specialist's efforts to a great extent using different interaction strategies - with normal and high tone, with positive or negative language.

Similar thesis is confirmed within the rest of variables results that demonstrate correlation without confirming the clear influence of linguistic or paralinguistic signs: lack of empathy in the use of negative language with high tone reported inverse correlation with insecurity in negative language with normal tone $(r=-.40 ; p<.001)$ and with insecurity in positive language with normal tone $(r=-.46 ; p<.001)$. Reverse correlation has also been registered 
within inability to seek help variable by the use of high tone and positive language with dependence variable by negative language with normal tone $(r=-.41 ; p<.001)$; as well as within inadequacy in positive language and high tone and dissatisfaction variable in negative language with normal tone $(r=-.41 ; p<.001)$. Data analysis does not clearly identify a trend within variables in any of the four strategies but an interesting dependence has been observed between interpersonal anxiety and self-assessment anxiety manifestations.

The child would be less empathic to others when negative words with raised tone are used but their insecurity about negative or encouraging language with normal tone would be reduced. Similar reverse dependence has been assigned in the use of encouraging language with high tone - the more independent and self-satisfied the child is, the less likely it is they would seek help and would respond more adequately without attracting attention in inappropriate way when negative language with normal tone is used. Therefore, empathy, seeking help, and adequacy as interpersonal relationship features are inversely related to self-reliance, independence and satisfaction as a projection of self-esteem and self - assessment. However, the more insecure, dependent, and dissatisfied a child is, the more empathy he or she would express to others, and they wouldn't attract attention in any way, nor would they know how to seek help when negative language in a normal tone is used. Present tendency could be explained by the self-assessment anxiety and interpersonal anxiety features - the more anxious an individual is, the more dependent on external stimulus they should be, and would be frustrated easily by outside sources of negative experiences respectively on the evaluation of others (indirect expression of which is empathy) and so individual would seek help from others and would avoid drawing attention in any case as there is a risk of rejection (Prihojan, 2000).

Inverse correlation has also been identified between reliance variable in the use of positive language with normal tone and dissatisfaction within negative language with normal tone $(r=-.68 ; p<.001)$, i.e. the more we use negative language with a normal tone, the less satisfied the child would be but applied strategy of the positive language with a high tone would increase his / her desire for independence.

Results outline additional nuances in terms of anxiety as a personal trait that does not always have a negative effect on child development, and certain anxiety indicators could interact each other in order to enhance social skills in the context of the proposed behavioral strategies - the negative and positive language combination with a different tone in different situations would increase social functioning of the child.

Therefore, reprimand and language imposing sanctions used by parents and specialists should not be denied at all costs, because it could as well lead to good results for the overall development of adolescents. The efficiency of different strategy combinations is further established in the present study.

Dynamics and impact of the four intervention strategies used is of particular interest in the present study. To this end, the results need to be analyzed more deeply in regard to individual combinations between linguistic signs (positive and negative language) and paralinguistic cues (high and normal tone).

Outliers of all ten anxiety variables were assigned by Student t-distribution for hypothesis test to assess the significance of the results (Table 3 ) and all four strategies - positive and negative language with high and normal tone register significant values $(p<0.05)$.

Table 3. Mean Values and t-distribution of 4 Strategies for Self-Assessment and Interpersonal Anxiety

\begin{tabular}{lccccccccc}
\hline & \multicolumn{7}{c}{ Self-assessment anxiety } \\
\hline & Mean & $\begin{array}{c}\text { Std. } \\
\text { Dv. }\end{array}$ & N & Std. Err. & $\begin{array}{c}\text { Coference - } \\
\text { Constant }\end{array}$ & t-value & df & $\mathrm{p}$ \\
\hline & & & & & & & & & \\
Positive language Normal tone & 0.37 & 0.93 & 40 & 0.146268 & 0 & 2,56379 & 39 & 0.01 \\
Positive language High tone & 0.27 & 0.72 & 40 & 0.113157 & 0 & 2,43025 & 39 & 0.01 \\
Negative language Normal tone & 2.48 & 1.32 & 40 & 0.208743 & 0 & 11,85667 & 39 & 0.00 \\
Negative language High tone & 2.35 & 1.56 & 40 & 0.246904 & 0 & 9,51787 & 39 & 0.00 \\
Positive language Normal tone & 0.65 & 1.09 & 40 & 0.173759 & 0 & 3,74081 & 39 & 0,000589 \\
Positive language High tone & 0.40 & 0.63 & 40 & 0.100000 & 0 & 4 & 39 & 0,000274 \\
Negative language Normal tone & 3.25 & 1.00 & 40 & 0.159124 & 0 & 20,4243 & 39 & 0 \\
Negative language High tone & 2.93 & 1.47 & 40 & 0.233116 & 0 & 12,54742 & 39 & 0 \\
\hline
\end{tabular}


Data analysis of mean values outline that negative language with a normal tone has higher impact on interpersonal and self-assessment anxiety of children, which does not confirm the hypothesis of the study assuming higher influence of the lexical signs with negative semantic meaning and high tone. Overall, the mean values of interpersonal anxiety indicators are much higher than self-assessment anxiety variables with less difference between negative language and high and normal tone. Negative language with normal tone reported stronger influence to interpersonal anxiety which could lead to the assumption that language and voice have higher impact on anxiety related to interpersonal relationships than anxiety as a self-assessment. Results strongly confirm the thesis of a direct correlation between negative stimulus and anxiety but voice power as a paralinguistic sign has an additional amendment that needs to be researched in depth.

"Var6"; LS Means

Wilks lambda $=, 61946, F(4,31)=4,7608, p=, 00412$

Effective hypothesis decomposition

Vertical bars denote 0,95 confidence intervals

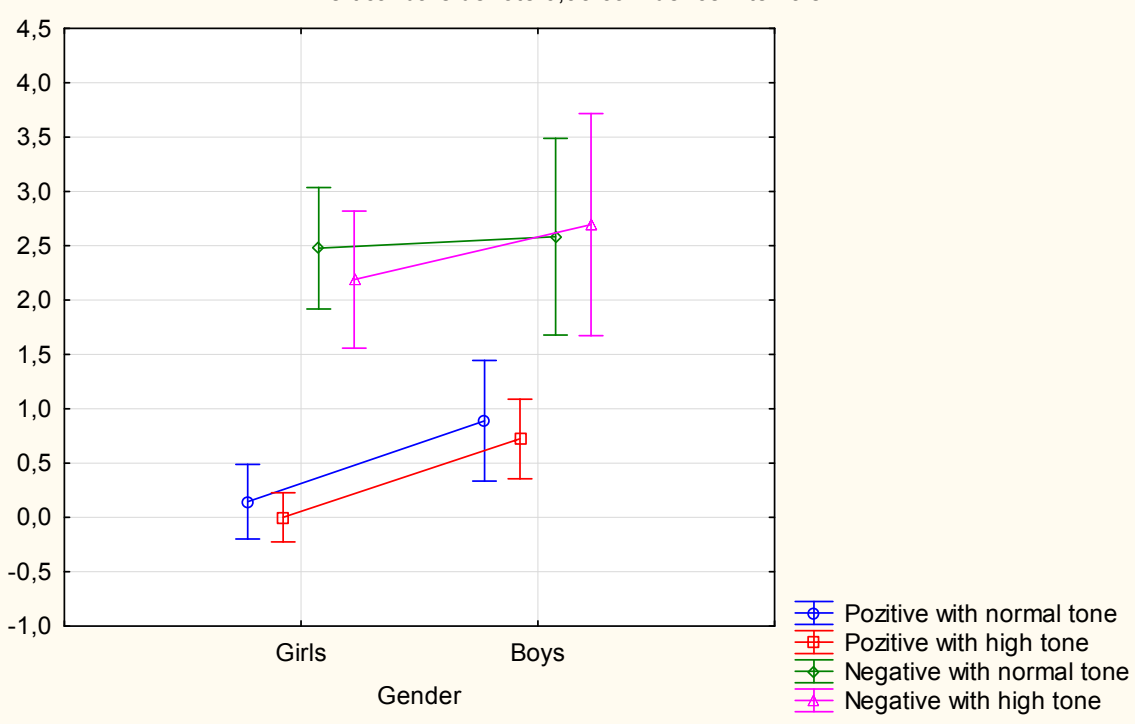

Figure 3. Gender Interaction in Self-Assessment Anxiety

ANOVA analysis of variances for the four strategies applied does not report significance of residential centre and age variables for interpersonal anxiety $(\mathrm{p}>.05)$ but self-assessment anxiety demonstrates significant differences in gender variable (Figure 3) and boys register increased anxiety in behavior in the use of positive language with normal tone $(F(1,34)=5.3768 ; p=0.02)$ and positive language with high tone $(F(1,34)=11.632 ; p=0.01)$. No significant differences have been reported between boys and girls in negative language with high and normal tone for self-assessment anxiety.

Age variable $(F(2,34)=3.7677 ; p=0.03)$ reported significant effect in interpersonal anxiety indicators with the use of negative language and high tone demonstrated in ANOVA analysis, as 7-10 years old and 15-17 years old participants register higher anxiety in comparison with 11-14 years old mates (Figure 4). All of the remaining independent variables such as gender, age and residential centre and their interaction did not report significance of interpersonal anxiety as well as self-assessment anxiety. 


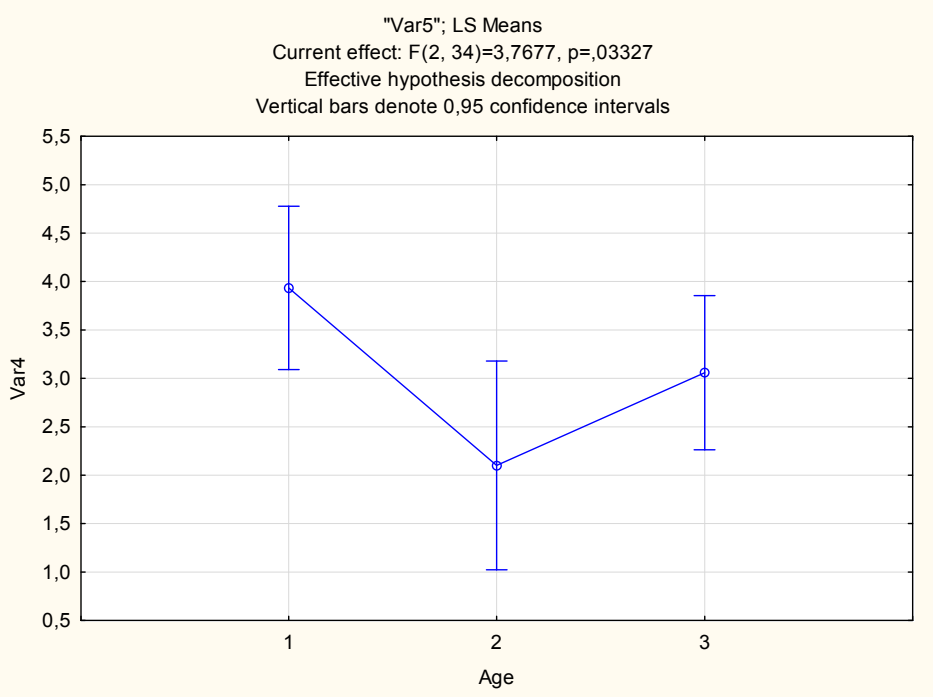

Figure 4. Age Interaction in Interpersonal Anxiety with the Use of Negative Language and High Tone

Correlation analysis does not report high relation between four intervention strategies neither for self-assessment anxiety, nor for interpersonal anxiety $(r<.04)$. Dynamics between self-assessment anxiety and interpersonal anxiety indicators register very high correlation in positive language with normal tone $(r=.81 ; p<.001)$; positive language with high tone $(r=.65 ; p<.001)$ and negative language with high tone $(r=.65 ; p<.001)$. Interaction between the four strategies in self-assessment anxiety and interpersonal anxiety in negative language and normal tone reported weaker correlation $(r=.44 ; p<.001)$. Data analysis assigns direct proportional dependence between two types of anxiety within the four strategies and it is rather influenced by anxiety as a personal trait of children than by intervention strategies impact.

Inverse correlation has also been identified between negative language with high tone strategy within interpersonal anxiety and negative language with normal tone at self-assessment anxiety $(r=-.51 ; p<.001)$. Similar pattern emerged in using negative language with normal tone within self-assessment anxiety and positive language with high tone for interpersonal anxiety $(r=-.47 ; p<.001)$. Results outline the assumption that self-assessment anxiety would increase when negative language with high tone is used but interpersonal anxiety would decrease within negative language with normal tone strategy.

In other words, the higher the child's self-assessment anxiety is, the more secure in interaction with others he/she would be, and the child would increase his/her anxiety when interacting with others in case he/she has low anxiety as a personal trait. The present trend has been traced out within the four strategies but their impact on behavior manifestations of intervention group needs to be investigated with another research taking into account personal traits or accentuation in children personality in order to avoid limitations.

A second intervention group has been randomly defined to test the hypothesis of the survey because ANOVA analysis does not register significance of residential centre independent variable in both self-assessment anxiety $(F(12,87,601)=1.2518 ; p=0.26)$ and interpersonal anxiety $(F(12,87,601)=1.2767 ; p=0.24)$. Eleven children from two of the residential centers were assessed by measuring their anxiety by means of questionnaires and specialists looking after them on daily basis.

Table 4. Mean Values of Self-assessment Anxiety and Interpersonal Anxiety of the Second Intervention Group

\begin{tabular}{lcclcc}
\hline Self-assessment anxiety & \multicolumn{5}{c}{ Interpersonal anxiety } \\
\hline & Mean & Std.Dv. & Mean & Std.Dv. \\
\hline Diffidence & 3.19 & 0.45 & Inadequacy & 2.92 & 0.88 \\
Dependence & 3.06 & 0.53 & Inactivity & 2.93 & 0.43 \\
Dissatisfaction & 2.85 & 0.54 & Non-communication & 2.37 & 0.72 \\
Reliance & 2.83 & 0.43 & Inability to seek help & 2.64 & 0.58 \\
Insecurity & 2.68 & 0.60 & Lack of empathy & 2.53 & 0.48 \\
\hline
\end{tabular}


Data analysis exhibit relatively high self-assessment anxiety and interpersonal anxiety based on their behavior in their usual environment. Very high mean values have been registered for diffidence and dependence variables which support the assumption from the first intervention group with 4 strategies that experienced children have very high anxiety levels as a personal trait. However, the intervention with four language strategies registered higher impact on their interpersonal anxiety while data collection from their usual behavior in a normal environment without external stimuli rather reported overwhelming self-assessment anxiety (Table 4).

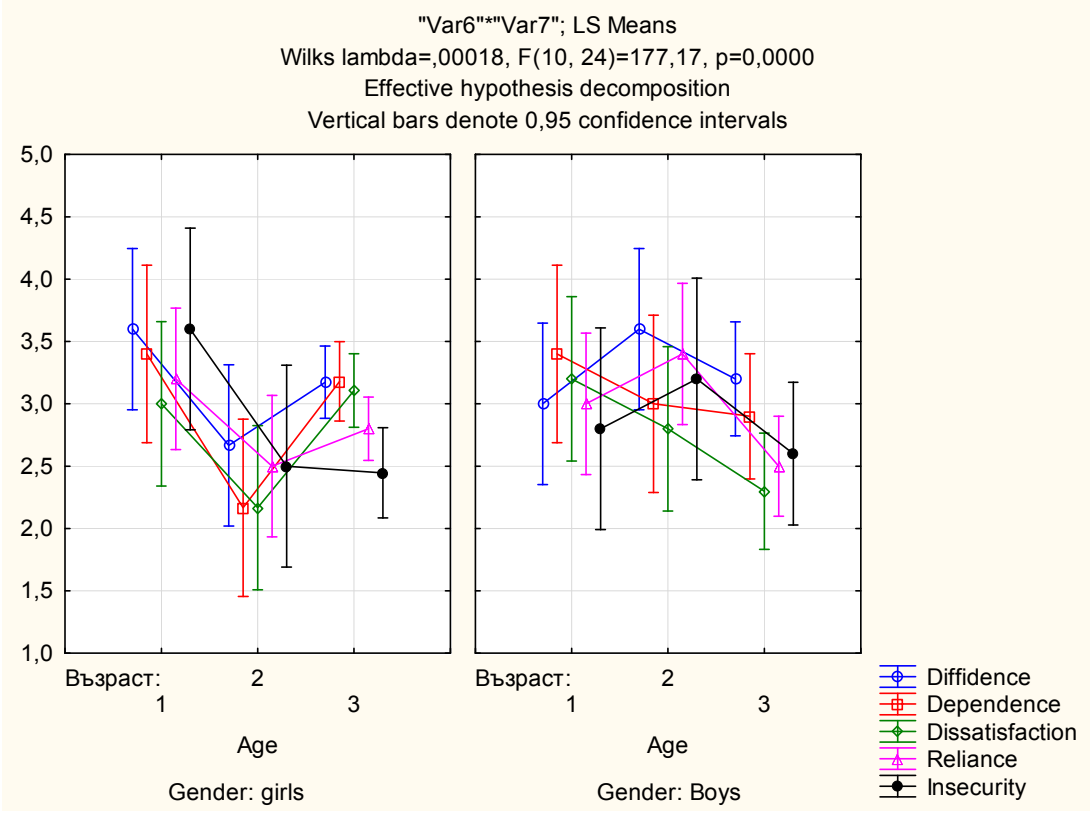

Figure 5. ANOVA Analysis of Gender and Age Variables Interaction

ANOVA analysis of variance for self-assessment anxiety reported significance for gender $(F(5,12)=632.28 ; p=$ $0.00)$ and age $(F(10,24)=49.436 ; p=0.00)$ as well as interaction between them $(F(10,24)=177.17) ; p=0.00)$ which demonstrates higher anxiety scope among 11-14 years old and 7-10 years old boys as well as 7-10 years old and 15-17 years old girls (Figure 5).

Correlation analysis demonstrates very high dependence between all 5 interpersonal anxiety variables with values around $r>.70 ; \mathrm{p}<.001$. Non communication reported very high dependence with inadequacy $(r=.87 ; p<.001)$, as well as with lack of empathy $(r=.6983 ; p<.001)$. Similar pattern emerged for inability to seek help with inactivity $(r=.74 ; p<.001)$ and with lack of empathy $(r=0.78 ; p<.001)$. Presented data suggests that the more problems with communication a child has, the more attention in an inappropriate way he/she would seek without considering the needs of others and the inability to seek help in cases of difficulty.

Self-assessment anxiety indicators demonstrate best correlation between dependence and dissatisfaction $(r=.89 ; p$ $<.001)$; reliance with diffidence $(r=.81 ; p<.001)$, insecurity $(r=.78 ; p<.001)$ and dissatisfaction $(r=.73 ; p$ $<.001)$; as well as insecurity with diffidence $(r=.71 ; p<.001)$. Data analysis indicate that lack of independence would increase the anxiety of the child when he/she does not receive encouragement or positive feedback and the child would also have problems in decision making (insecurity) as well as in being persistent in difficult situations.

The impact of self-assessment and interpersonal anxiety is tracked by the correlation analysis between their individual indicators. Reliance demonstrates very high correlation with inability to seek help $(r=.84 ; p<.001)$ as well as with lack of empathy $(r=.87 ; p<.001)$. High dependence demonstrates diffidence with non-communication $(r=.86 ; p<.001)$ inactivity $(r=.71 ; p<.001)$ and inability to seek help $(r=.74 ; p<.001)$; and inadequacy reports very good relation with diffidence $(r=.71 ; p<.001)$, dependence $(r=.77 ; p<.001)$ and dissatisfaction $(r=.75 ; p$ $<.001)$. Insecurity registers good correlation with inability to seek help $(r=.70 ; p<.001)$ and lack of empathy $(r$ $=.70 ; p<.001)$. Present data analysis could lead to the assumption that diffidence, lack of self-esteem and self-dependence would influence relationships with others in greater extent and the child's integration into a group and society would be affected according to its adequacy in situations, ability to seek help in difficult situations as 
well as how much the child respects the other people's needs. Therefore, communication skills as a qualitative feature of interpersonal anxiety are greatly enhanced by self-assessment anxiety as a personal accent and trait. Additionally, some differences have been identified between anxiety behavioral expressions within the experienced four strategies and personal characteristics of the studied children with regard to their anxiety manifestations in daily life evaluated by specialists. The experimental impact digests that the more confident the child is, the less empathetic to others he/she would be when negative language with high tone is used, while the general assessment of specialists reported a reverse tendency and empathy towards others is more likely for more confident children. Presented contradiction assigns that the change in behavior should have been provoked by negative language with high tone influencing the anxiety of the child.

In-depth analysis of the four strategies impact on ten anxiety indicators requires further data decomposition as each of the 4 language-tone combinations is transferred to a 4 grade scale value that does not express quantity but indicates qualitative feature in order to distinguish strategies among them: positive language and normal tone - 1; positive language and high tone -2 ; negative language and normal tone -3 ; negative language and high tone -4 , being provisionally divided into a range of 0.7 points ( 1 - from 1 to 1,$7 ; 2$ - from 1,8 to 2,$4 ; 3$ - from 2,5 to 3,2 and 4 - from $3,3$ to 4$)$.

Table 5. Mean Values and t-distribution for 4 Strategies among 10 Anxiety Indicators

\begin{tabular}{lcccccccc}
\hline & $\begin{array}{c}\text { Positive } \\
\text { language } \\
\text { Normal } \\
\text { tone }\end{array}$ & $\begin{array}{c}\text { Positive } \\
\text { language } \\
\text { High tone }\end{array}$ & $\begin{array}{c}\text { Negative } \\
\text { language } \\
\text { Normal } \\
\text { tone }\end{array}$ & $\begin{array}{c}\text { Negative } \\
\text { language } \\
\text { High tone }\end{array}$ & Mean & Std.Dev. & t-value & $p$ \\
\hline Diffidence & $10 \%$ & $7,5 \%$ & $45 \%$ & $37,5 \%$ & 3.10 & 0.93 & 21,1229 & 0.0000 \\
Dependence & $2,5 \%$ & $7,5 \%$ & $55 \%$ & $35 \%$ & 3.23 & 0.70 & 29,2416 & 0.0000 \\
Dissatisfaction & $0 \%$ & $7,5 \%$ & $55 \%$ & $37,5 \%$ & 3.30 & 0.61 & 34,3475 & 0.0000 \\
Reliance & $0 \%$ & $10 \%$ & $55 \%$ & $35 \%$ & 3.25 & 0.63 & 32,6047 & 0.0000 \\
Insecurity & $0 \%$ & $2,5 \%$ & $60 \%$ & $37,5 \%$ & 3.35 & 0.53 & 39,7142 & 0.0000 \\
Inadequacy & $15 \%$ & $2,5 \%$ & $40 \%$ & $42,5 \%$ & 3.10 & 1.03 & 18,9836 & 0.0000 \\
Inactivity & $15 \%$ & $15 \%$ & $47,5 \%$ & $22,5 \%$ & 2.78 & 0.97 & 18,0248 & 0.0000 \\
Non-communication & $0 \%$ & $2,5 \%$ & $60 \%$ & $37,5 \%$ & 3.35 & 0.53 & 39,7142 & 0.0000 \\
Inability to seek help & $5 \%$ & $2,5 \%$ & $50 \%$ & $42,5 \%$ & 3.30 & 0.76 & 27,5393 & 0.0000 \\
Lack of empathy & $0 \%$ & $2,5 \%$ & $55 \%$ & $42,5 \%$ & 3.40 & 0.55 & 39,4287 & 0.0000 \\
\hline
\end{tabular}

Data collection outlines that negative language with normal tone has an impact to self-assessment and interpersonal anxiety of children to a greater extent while negative language with high tone increases anxiety of intervention group only for inadequacy variable (table 6). Comparative analysis of frequency distribution and mean values of negative language with high and normal tone assigns emphatically stronger impact of the normal tone. Data collection outlines that negative language with high tone largely influences interpersonal anxiety variables as non communication, inability to seek help and lack of empathy evident in Table 6. Self-assessment anxiety variables insecurity and dissatisfaction are also enhanced by negative language with high tone although they show a significantly higher increase from negative language with a normal tone.

ANOVA analysis of independent variable diagnosis of second intervention group $(N=11)$ also reported significance of self-assessment anxiety $(F(4,17)=5.2183 ; p=0.00)$, and interpersonal anxiety $(F(4,17)=5.1051 ; p=0,00)$, although surveyed children having diagnosis (emotional, behavioral and personality disorders) were almost twice less than others. Results outline that self-assessment and interpersonal anxiety of children with emotional disorders are mostly increased by negative language with high tone, while negative language with normal tone reported lower impact on their interpersonal anxiety than other children (Figure 6). 


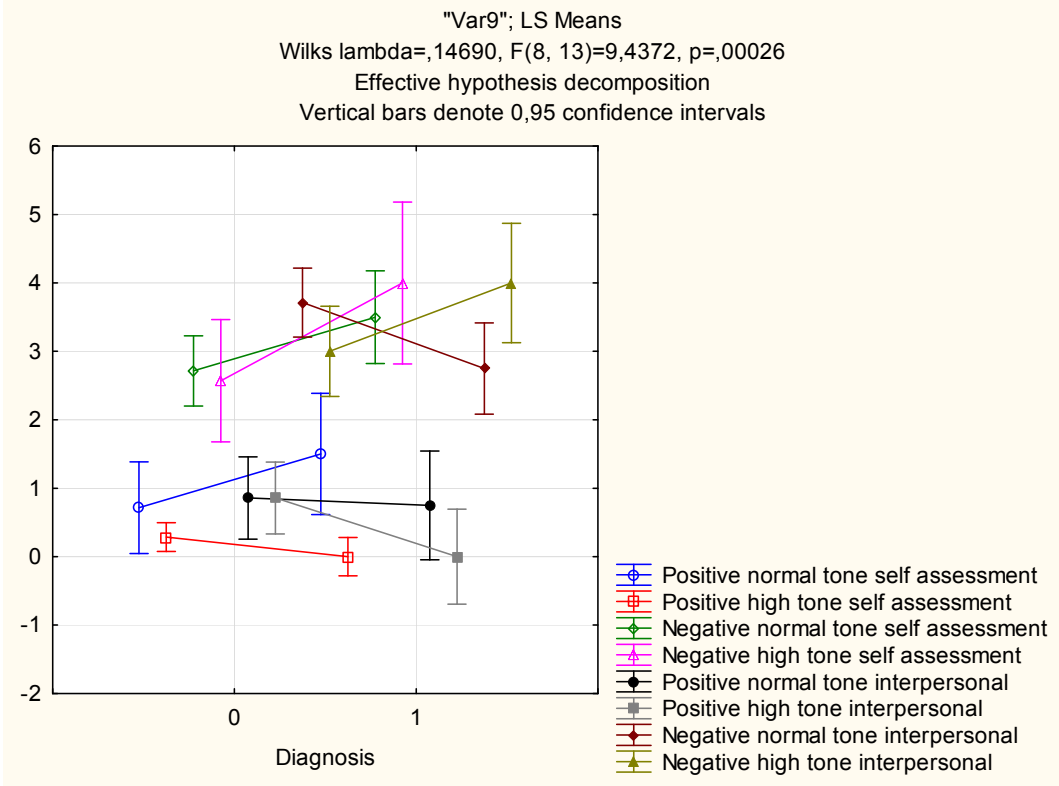

Figure 6. ANOVA Analysis of Diagnosis on Self-Assessment Anxiety and Interpersonal Anxiety

Comparative analysis of the results of both intervention experiments for only the re-examined group of children assigns that negative language with normal tone has a higher impact on dependence from external stimuli as a reverse correlation has been reported between these two variables $(r=-.47 ; p<.001)$. Reported results could lead to the assumption that negative language with normal tone as a strategy would increase the independence of the child. A completely different tendency has been outlined with regard to interpersonal anxiety as negative language with normal tone does not register any significant influence to it.

There is a completely different tendency with regard to interpersonal anxiety - negative language with normal tone does not have any statistically significant effect opposed to negative language with high tone that reported dependence with inadequacy $(r=.50 ; p<.001)$ and non communication $(r=.49 ; p<.001)$ indicating that increasing the tone would also increase the inadequate reactions and communication of the child. Positive language with high tone also demonstrates good correlation with inactivity $(r=.43 p<.001)$, in its quality as an interpersonal anxiety variable confirming the hypothesis of negative impact of the tone on the child's anxiety in their relationships with others.

Comparative analysis between mean values of all 10 anxiety indicators of the second re-examined group (N=11) in the first intervention with four strategies assigns that only negative language with normal tone reports correlation of total anxiety in the children $(r=.4410 ; p<.001)$. Therefore, it has been acknowledged the strongest impact of negative language with normal tone which does not confirm the general hypothesis of the present study but enhance strong reasons to assume that proper use of negative language with normal tone would affect acquisition of social skills among the studied group of children.

\section{Discussion}

The study strongly confirms the presumption of a direct dependence between negative stimulus and anxiety but voice power as a paralinguistic sign outlines an additional influence in accordance with individual features and personality of children. Data analysis suggests that extent of activity involvement of the child as well as independence, confidence and security rather depends on anxiety as a personal trait of the child, and is not that influenced by the specialist efforts using different interaction strategies - with normal and high tone, with positive or negative language. Results strongly confirm the thesis of a direct correlation between negative stimulus and anxiety and tone as a paralinguistic sign has an additional amendment.

Scientific findings of this study outline that insecurity; diffidence and dependence most affect relationship with others and integration of the child into the group and society would be affected according to their adequacy in situations, empathy to others and ability to seek help in difficult cases. Therefore, communication skills as a 
qualitative feature of interpersonal anxiety are greatly enhanced by self-assessment anxiety as a personal accent and trait. Additionally, the experimental impact digests that the more confident the child is, the less empathetic to others he/she would be when negative language with high tone is used, while in a situation without external source of negative experiences, empathy for others is more inherent in children who are more confident in themselves. Therefore, empathy, seeking help, and adequacy as interpersonal relationships features are inversely related to self-reliance, independence and satisfaction as a projection of self-esteem and self-assessment. This assumption could be explained by the self-assessment anxiety and interpersonal anxiety features - the more anxious an individual is, the more dependent on external stimulus he/she should be, and would be frustrated easily by outside sources of negative experiences respectively on the evaluation of others and, thus an individual would seek help from others and would avoid drawing attention in any case as there is a risk of rejection (Prihojan, 2000). Therefore, the higher child self-assessment anxiety is the more secure in interaction with others they would be, and would increase anxiety of the child when interacting with others in case he/she has low anxiety as a personal trait.

This study assigns that the change in behavior should have been provoked by negative language with high tone influencing self-assessment anxiety of the child but interpersonal anxiety would be decreased within negative language with normal tone strategy.

Self-assessment and interpersonal anxiety in children with emotional disorders are mostly increased by negative language with high tone, while negative language with normal tone reported lower impact on their interpersonal anxiety than in other children.

However, it has been acknowledged that the strongest impact of negative language with normal tone which does not confirm the general hypothesis of the present study but enhance strong reasons to assume that proper use of negative language with normal tone would affect acquisition of social skills among the studied group of children. Therefore, balanced use of reprimand and sanctioning language with normal tone used by parents and specialists should not be denied at all costs, but would as well lead to good results in the overall development of children.

\section{Conclusion}

Results outline additional nuances in terms of anxiety as a personal trait that does not always have a negative effect on the child's development, and certain anxiety indicators could interact with each other in order to enhance social skills in the context of the proposed behavioral strategies - the negative and positive language combination with a different tone in different situations would increase the social functioning of the child.

The use of different strategies for interaction by the specialist - with normal and high tone, encouragement or reprimand should be tailored to the specifics of the child as well as to the relevant skills to be developed. The scientific findings of this study could support teachers, social workers, psychologists and further specialists to find an appropriate communication strategy to reduce anxiety in order to enhance personal development and socialization process of children. The study could provide some guidance for an effective educational and counseling model to support the efforts of social workers, psychologists, and teachers to find an appropriate strategy for communication and proper language use for supporting development of children. Further in-depth research is needed about how the use of language would influence child behavior and development.

\section{Acknowledgements}

This research was supported by National Science Fund of Ministry of Education and Science, Bulgaria, Innovative approaches for developing social functioning of children and youth project, Contract № DM 15/3, 20.12.2017. Thank you to ZOV UK charity and Veliko Tarnovo Municipality for the opportunity to complete the survey.

\section{References}

Barlow, D. (2002). Anxiety and Its Disorders. The nature and treatment of anxiety and panic. New York: The Guilford Press ISBN: 1-59385-028-X

Clarkson, M. G., \& Clifton, R. K. (1985). Infant pitch perception: evidence for responding to pitch categories and the missing fundamental. Journal of the Acoustical Society of America, 77, 1521-1528. https://doi.org/10.1121/1.391994

Dimitrova, Y. (2016). Specifics of social work with different at-risk groups. Papers from the Scientific Conference "Social Service in Contemporary Society" - Veliko Tarnovo: Libra - Scorp, 189-196. 
Doncheva, K. Z. (2019). Language Impact towards Social Behavior of At-Risk Children, PUPIL: International Journal of Teaching, Education and Learning, 3(1), 01-21. https://dx.doi.org/10.20319/pijtel.2019.31.0121

Friend, M. (2000). Developmental changes in sensitivity to vocal paralanguage. Developmental Science, 3, 148-162. https://doi.org/10.1111/1467-7687.00108

Friend, M. (2003). What Should I Do? Behavior Regulation by Language and Paralanguage in Early Childhood. Journal of Cognition and Development, 4, 161-183. https://dx.doi.org/10.1207/S15327647JCD0402_02

Friend, M., \& Bryan, J. B. (2000). A developmental lexical bias in the interpretation of discrepant messages. Merrill-Palmer Quarterly, 46, 140-167.

Georgiev, G. (2019) Sociopedagogical aspects of work with delinquent children. V. Tarnovo: IVIS Edition, 250.

Greca, A., \& Lopez, M. (1998). Social anxiety among adolescents: Linkages with Peer Relations and Friendships. Journal of Abnormal Child Psychology, 26(2), 83-94. https://doi.org/10.1023/A:1022684520514

Hancock, J. T., Dunham, P. J., \& Purdy K. (2000). Children's comprehension of critical and complimentary forms of verbal irony. Journal of Cognition and Development, 1, 227-248. http://dx.doi.org/10.1207/S15327647JCD010204

Kitamura, C., \& Burnham, D. (2003). Pitch and Communicative Intent in Mother's Speech: Adjustments for Age and Sex in the First Year. Infancy, 4(1), 85-110. https://doi.org/10.1207/S15327078IN0401_5

Kitamura, C., \& Lam, C. (2009). Age-Specific Preferences for Infant-Directed Affective Intent._Infancy, 14, 77-100. https://doi.org/10.1080/15250000802569777

Kuteva-Tsvetkova, V. (2000). Problems of family pedagogy. V. Tarnovo: University Edition "St. Cyril and St. Methodius".

Kuzzmanova-Kartalova, R. (2013). Theoretical concepts and methodic approaches in sociopedagogical work. V. Tarnovo: University Edition "St. Cyril and St. Methodius".

Leary, M. R. (1983). Understanding social anxiety: Social, personality, and clinical perspectives. Beverly Hills, C. A: Sage.

Moore, C., Harris, L., \& Patriquin, M. (2008). Lexical and prosodic cues in the comprehension of relative certainty. Journal of Child Language, 20, 153-167. https://doi.org/10.1017/S030500090000917X

Moore, K. A., Vandivere, S., \& Redd, Z. (2006). A Sociodemographic Risk Index. Social Indicators Research Series, 27, 45-81. https://doi.org/10.1007/s11205-004-6398-7

Morton, J. B., \& Trehub, S. E. (2001). Children's understanding of emotion in speech. Child Development, 72, 834-843. https://doi.org/10.1111/1467-8624.00318

Prihojan, A. M. (2000). Anxiety among children and adolescents: Psychological concepts and age dynamics. Psychology and social institute of Moscow; Voronej: NPO "MODEK" Edition.

Rak, C. F., \& Patterson, L. E. (1996). Promoting resilience in at-risk children. Journal of Counseling and Development, 74(4), 368-373. http://dx.doi.org/10.1002/j.1556-6676.1996.tb01881.x

Solomon, D., \& Ali, F. A. (1972). Age trends in the perception of verbal reinforcers. Developmental Psychology, 7 , 238-243. https://doi.org/10.1037/h0033354

Stamatov, R. (2000). Child psychology. Plovdiv: HERMES Editor, Plovdiv.

Zdravkova, B. (2015). Sociopedagogical work with children dropped out of school. Proceedings of the Jubilee International Scientific Conference "30 Years of the Faculty of Education". V. Tarnovo: Faber, 142-147. ISBN 978-619-00-0352-6

Zlatkova-Doncheva, K. (2017). Effect of Mentoring at-risk youth program in Bulgaria. Berlin. LAP LAMBERT Academic Publishing, 2017, ISBN: 978-620-2-01891-3

Zlatkova-Doncheva, K. (2018). Mentoring and children at risk. LIBRA SCORP, 288p. ISBN: 978-954-471-438-3. 